\title{
AnnotatEd: A Social Navigation and Annotation Service for Web-based
} Educational Resources

\author{
Rosta Farzan $^{\mathrm{a} *}$ and Peter Brusilovsky ${ }^{\mathrm{b}}$ \\ ${ }^{a}$ Intelligent Systems Program, University of Pittsburgh, 210 S. Bouquet St., Pittsburgh, 15260, \\ USA, phone: +1(412) 624 5757, fax: +1 (412) 6242788 , \\ rosta@cs.pitt.edu, ; \\ ${ }^{\mathrm{b}}$ School of Information Sciences, 135 North Bellefield Avenue, University of Pittsburgh, \\ Pittsburgh, 15260, phone: +1 (412) 624 9404, fax: +1 (412) 6242788 \\ peterb@mail.sis.pitt.edu,USA
}

* Corresponding author 
AnnotatEd: A Social Navigation and Annotation Service for Web-based Educational Resources 


\begin{abstract}
Web page annotation and adaptive navigation support are two active, but independent research directions focused on the same goal: expanding the functionality of the Web as a hypertext system. The goal of the AnnotatEd system presented in this paper has been to integrate annotation and adaptive navigation support into a single value-added service where the components can reinforce each other and create new unique attributes. This paper describes the implementation of AnnotatEd from early prototypes to the current version, which has been explored in several contexts. We summarize some lessons we learned during the development process and which defined the current functionality of the system. We also present the results of several classroom studies of the system. These results demonstrate the importance of the browsing-based information access supported by AnnotatEd and the value of both the annotation and navigation support functionalities offered by the system.
\end{abstract}

Keywords: Web Annotation; Social Navigation; E-Learning; Navigation Support; Evaluation; Classroom Study

\title{
1 Introduction
}

The World Wide Web is currently the single most popular hypertext system; however, it is far from being the most powerful or the most advanced hypertext system. A number of the advanced features implemented in various pre-Web hypertext systems have been "lost" in the move from classic hypertext to the Web. As a result, a number of international research groups are now working on extending Web hypertext so that it will have the advanced functionalities which were already known to be valuable for pre-Web hypertext users.

Content annotation is among the most active branches of work that is focused on enhancing the Web as a hypertext system. A number of pre-Web hypertext systems supported annotation in the form of highlighting or underlining a part of the text, or associating free format or structured comments with a portion of a page. The ability to annotate is important, but it is not inherent to Web architecture. To compensate for the missing functionality, many Web-based closed-corpus hypertext systems (such as virtual museums) offered proprietary tools for the users to annotate their own content. In the open-corpus context (unrestricted Web content), the ability to annotate was originally supported on the client side by some early browsers, but later became a function found within a range of Web annotation systems that provide value-added services and store annotation on the server side. The move from client-side to server-side annotation was originally caused by browser problems but was later fuelled by the extra value provided by centrally storing annotations: the ability to share annotations with others (Gibeo, 2004; Xin and Glass, 2005). This ability enables open and closed-corpus Web annotation tools to support collaborative work over the Web.

Other directions of work that are focused on enhancing the Web with advanced hypertext functionality include guided tours and adaptive navigation support. Guided tours allow a user to navigate a complex hyperspace along a linear, predefined path, which is frequently augmented with a narration. This functionality was implemented in several closed-corpus Web hypertext systems. To offer guided tours on the open-corpus Web, both browser-side support (Moody, 1998; Noda et al., 2004) and intermediary-style systems (Sandvad et al., 2001; Shipman et al., 2000) were suggested. Adaptive navigation support helps the user select the most relevant links 
to navigate. It has been implemented in many closed-corpus adaptive hypermedia systems (Brusilovsky, 2001) and a few open-corpus systems (Pazzani et al., 1996).

When researching advanced hypertext functionality for Web browsing, one of the problems is how to support several functionalities in one system. While each enhancement offers some added value, existing projects focus on advancing Web hypertext in only one area whether it is annotation, guided tours, or navigation support. The most interesting research question is how to integrate two or more of these enhancements so that the result will be more than the sum of its parts. For example, the integration of annotation and guided tours could enhance the value of guided tours by including both author- and user-created narrations (Shipman et al., 2000).

The goal of the project presented in this paper was to integrate annotation and adaptive navigation support for open-corpus Web hypertext into a single value-added service. This paper introduces the AnnotatEd system, which offers both annotation (through highlighting and freetext comments) and adaptive navigation support (through social navigation). It works in the traditional intermediary way (Barrett and Maglio, 1999), standing between Web pages and users. Each Web page is pre-processed by AnnotatEd before being displayed to the user. During this pre-processing stage, AnnotatEd adds adaptive visual cues to all eligible links and generates an additional annotation frame that allows the user to view and provide annotation. The navigation support and annotation components in AnnotatEd enhance each other's value. The navigation support component makes the user's annotations more visible and valuable by adding visual cues to all links that lead to pages previously annotated by the user. In turn, access to annotations written by multiple users on the same pages allows the social navigation support component to extend the traditional traffic-based support with annotation-based support.

This presentation is organized as follows: Sections 2 and 3 review related work in the area of Web annotation and social navigation. Section 4 gives an overview of the AnnotatEd system and describes the different stages it went through while being developed. Sections 5 and 6 present the internal mechanisms of AnnotatEd. Here we summarize the major development stages of these mechanisms and discuss the lessons learned. Section 7 reports the most important results found in our evaluation of AnnotatEd. Section 8 concludes the paper and describes some future directions for research.

\section{Web Annotation}

Web annotation has been popular among both researchers and practitioners. The list of Web annotation tools is growing every year. While practitioners have been mostly focused on providing more and more sophisticated interfaces with several annotation functionalities, the researchers have been more interested in exploring the additional benefits that can be provided in Web annotation systems. Accordingly, the review below distinguishes works focusing on the annotation interface from works providing annotation in a different context.

\subsection{Web Annotation Interfaces}

Several annotation systems focus on developing an advanced architecture and building a sophisticated but user-friendly annotation interface to improve Web-based annotations. They try to support content-aware annotation on any arbitrary Web page. Gibeo is an example of a welldesigned Web annotation system. Once the user is registered with their Website, the user may annotate any random Web page by merely adding ".gibeo.net" to the URL. When any part of the 
text on the page is highlighted, a set of options is displayed to allow the user to specify whether the highlighted text is important, wrong, or cool. The users can also add comments, corrections, links, or shared discussion to any part of the text. All the annotation is shared with all users of the system and clicking on an annotation provides detailed information about the annotation, such as the name of its author.

Marginalia (Xin and Glass, 2005) is a JavaScript Web annotation system that focuses on providing intuitive annotation functionality for any arbitrary Web page or comments made in the Moodle discussion forum. Marginalia allows users to highlight any part of the text and write associated comments in the margin of the pages. Annotation may be marked as public or private.

CritLink (Yee, 2002) is a Web annotation tool that allows annotation to any location on any public page, in order to support critical discussion and document annotation. It doesn't require installing any special client or server software and is supported by all browsers and operating systems. Annotations can have types as well as be public or private. CritLink augments the browsing experience by mediating all transactions between the browser and server. CritLink also tries to support other important hypertext functionalities such as bi-directional links, extrinsic links, typed links, and fine-grained links.

\subsection{Web Annotation and Collaborative Work}

The use of annotation for collaborative work is a popular branch of research that started well before the Web era. A range of important studies were performed with pre-Web annotation systems. For example, (Wojahn et al., 1998) studied the effect of an annotation interface on collaboration by comparing three different annotation interfaces. They concluded that annotation interfaces affect the number and types of problems about which collaborators communicate. These studies provided a foundation for developing collaborative annotations on the Web.

Arguably, the most well-known annotation tool in this category is the Annotea system, which enhances the collaborative development of the Semantic Web via shared Web annotations (Kahan et al., 2002). Annotations are in the form of comments, notes, explanations, or any other type of external remarks attached to any Web document or portion of the document. The users are able to access all attached annotations when they open the original document.

Microsoft Office 2000 includes a "Web discussion" feature that allows collaborative annotation of any Web page. (Cadiz et al., 2000) studied the application of the Microsoft Office $2000 \mathrm{Web}$ annotation tool for collaborative writing within a large product group at Microsoft. Cadiz et al provide a detailed analysis of the factors influencing the collaborative usage of the Microsoft 2000 tool. Their results indicated that there was quite a variation in the usage of these annotations.

MADCOW (Bottoni et al., 2006) is another example of a browser-based annotation tool for multimedia resources. MADCOW is designed to support document-centered collaboration. It offers context-bounded discussions and annotations of annotations.

\subsection{Web Annotation and Information Access}

Research on Web annotation for information access attempts to develop tools that use user annotation behavior to improve the user's ability to retain and retrieve relevant information. A landmark pre-Web study of this type was about XLibris, a pen-based freeform annotation tool that supports highlighting, underlining, and commenting. XLibris employs users' marks for the organization of information and to pinpoint information access by generating search queries from 
users' annotations. In addition, XLibris provides a skimming mode, which highlights the most important parts of the document retrieved, based on other users' annotations. It also processes the highlighted text and offers links to similar documents (Schilit et al., 1998). More recently, Bradshaw and Light studied the degree of overlap between annotations when different researchers read the same article (Bradshaw and Light, 2007). They found a high degree of overlap in the annotated passages and they concluded that the users' annotations are a reliable source for recommending important passages.

YAWAS is an example of a similar Web annotation tool that allows users to highlight any part of the text. It employs user annotation to provide personalized document summaries, by conglomerating the highlighted text. The highlighted portions of the documents can also be used for personalized document clustering and classification (Denoue and Vignollet, 2001).

\subsection{Web Annotation and E-Learning}

E-learning is an important domain that has benefited from Web annotation tools. EDUCOSM (Kurhila et al., 2003) is an example of how annotation tools are used in e-learning. It allows annotations in the form of highlights and comments on any Web page. EDUCOSM also supports collaborative knowledge building by allowing joint annotation of resources. All the annotations are visible to all students, which helps them to learn from each other.

(Rau et al., 2004) also developed a Web annotation tool that allows users to annotate Web-based resources and to share them with other learners. Annotations are in the form of highlighted texts and comments. The tool also allows the inserting and editing of annotations, and the ability to organize and present annotations hierarchically. Users have the option to keep the annotation individual or to share them with others. Sharing of annotations can be done synchronously or asynchronously.

WebAnn (Brush et al., 2002) is a shared annotation system, which is embedded into Internet Explorer and allows the annotation of any random Webpage. Annotations are contextdependent and can be associated with any part of the page. A study comparing WebAnn with a discussion board system in a graduate course showed that students made significantly more comments using WebAnn because of the ability to make contextual comments.

Web Memo (Yanagisawa et al., 2004) is a Web browser that allows students to annotate Web pages by highlighting, writing comments, and drawing with a freehand pen. Evaluation of this system among undergraduate students showed that the subjects were satisfied with the system. Moreover, the evaluation showed that the frequency of highlighting, which is the most frequent form of annotation, does not differ, whether using the Web Memo system or paperbased annotation.

\section{Social Navigation Support}

Adaptive navigation support is a group of interface techniques developed in the field of adaptive hypermedia (Brusilovsky, 2001) for guiding users to the most appropriate information resources. However, classic adaptive navigation support techniques rely on manual annotation produced by domain experts and are not well suited to structuring guidance for the large volume of open-corpus documents (Brusilovsky and Henze, 2007). Social navigation support (SNS) techniques provide an alternative solution, which is based on the ideas of social navigation (Dieberger et al., 2000). Dourish and Chalmers (1994) define social navigation as "moving towards clusters of people" or "selecting subjects because others have been examining them." 
SNS techniques make use of many users' past interactions with the system to guide new users of the system, relying on the collective knowledge of a large community of users. The kind of interaction past learners had is taken into account by a SNS system; we can distinguish several types of SNS. The two types of SNS provided in AnnotatEd are traffic-based and annotationbased SNS.

Traffic-based SNS originates from the traditional "footprint" approach to social navigation (Wexelblat and Mayes, 1999). It generally provides information about the number of visits users have made to each link. Traffic-based SNS promotes links which have a higher number of visits. For example, CoWeb extends a traditional WIKI hypertext by visualizing traffic-based social navigation (Dieberger and Guzdial, 2003). The system tracks how often a page is accessed or modified. It visualizes the density of the aggregated access for the past 24 hours by applying three levels of color intensity to the footprint symbol.

Annotation-based SNS is based on the annotation activities of the users, thus offering more reliable evidence of page importance than simple visitor count (the reference is removed for blind review). Annotation-based SNS promotes links to pages annotated by users, especially pages with higher numbers of annotations or positive annotations. Annotation-based SNS is less frequently used. EDUCO (Kurhila et al., 2002) provides an annotation tool which allows learners to associate comments with a document. It provides simple annotation-based SNS by keeping track of when comments are modified and visually demarcates new comments.

\section{The Interface of AnnotatEd: From Knowledge Sea II to ASSIST-ACM}

The AnnotatEd system presented in this paper is a social navigation and annotation service that employs users' annotation and navigation information to provide social navigation support. AnnotatEd allows students to annotate Web pages by placing free-format comments that can be associated with the whole page or specific parts of the page, which are highlighted. Similar to other intermediary-type systems (Barrett and Maglio, 1999), AnnotatEd accompanies the students from page to page by redirecting all the page links through the AnnotatEd proxy service. The proxy service pre-fetches requested pages and augments links inside the pages in order to offer social adaptive navigation support.

The navigation support is offered in a popular form-link annotation (Brusilovsky, 1996) and is personalized on both the group and individual level. AnnotatEd provides a visual cue next to each link, expressing the past interactions of the user and the group the user belongs to. To offer social navigation support, AnnotatEd keeps track of all the user's actions: every click they make, the time they spend reading each page, and every annotation they make for each page.

Figure 1 shows a sample page presented by AnnotatEd. As can be seen in the figure, the links inside the pages are augmented with traffic- and annotation-based visual cues. The algorithms to generate these visual cues are explained in sections 4.4 and 4.5 . The right side of the page is the annotation frame, which allows the user to add notes to the page, highlight a fragment of text, or bookmark a page. When adding a note, in addition to creating free-format text, the user has the option to make the note private or share it with others and specify a type for the note. The note can be signed with the user's name or be anonymous. To highlight a section, the user selects any part of the text and clicks on the highlight button. The user can also bookmark the page by clicking on the bookmark button and later retrieve the bookmarked pages by clicking on the summary button.

AnnotatEd is a multi-purpose system, which can be integrated into different Web-based systems, adding social navigation support to an existing system. In this section, we present two 
examples of using AnnotatEd to support student work with external educational resources. The first one is the integration of AnnotatEd with Knowledge Sea II, which attempted to help students find the most useful pages in Web-based textbooks and tutorials (Brusilovsky et al., 2004). The second example demonstrates the use of AnnotatEd in the ASSIST-ACM system (Farzan et al., 2007), which was designed to help students and researchers locate relevant articles in the electronic version of Communications of the ACM, a magazine available through ACM's Digital Library*

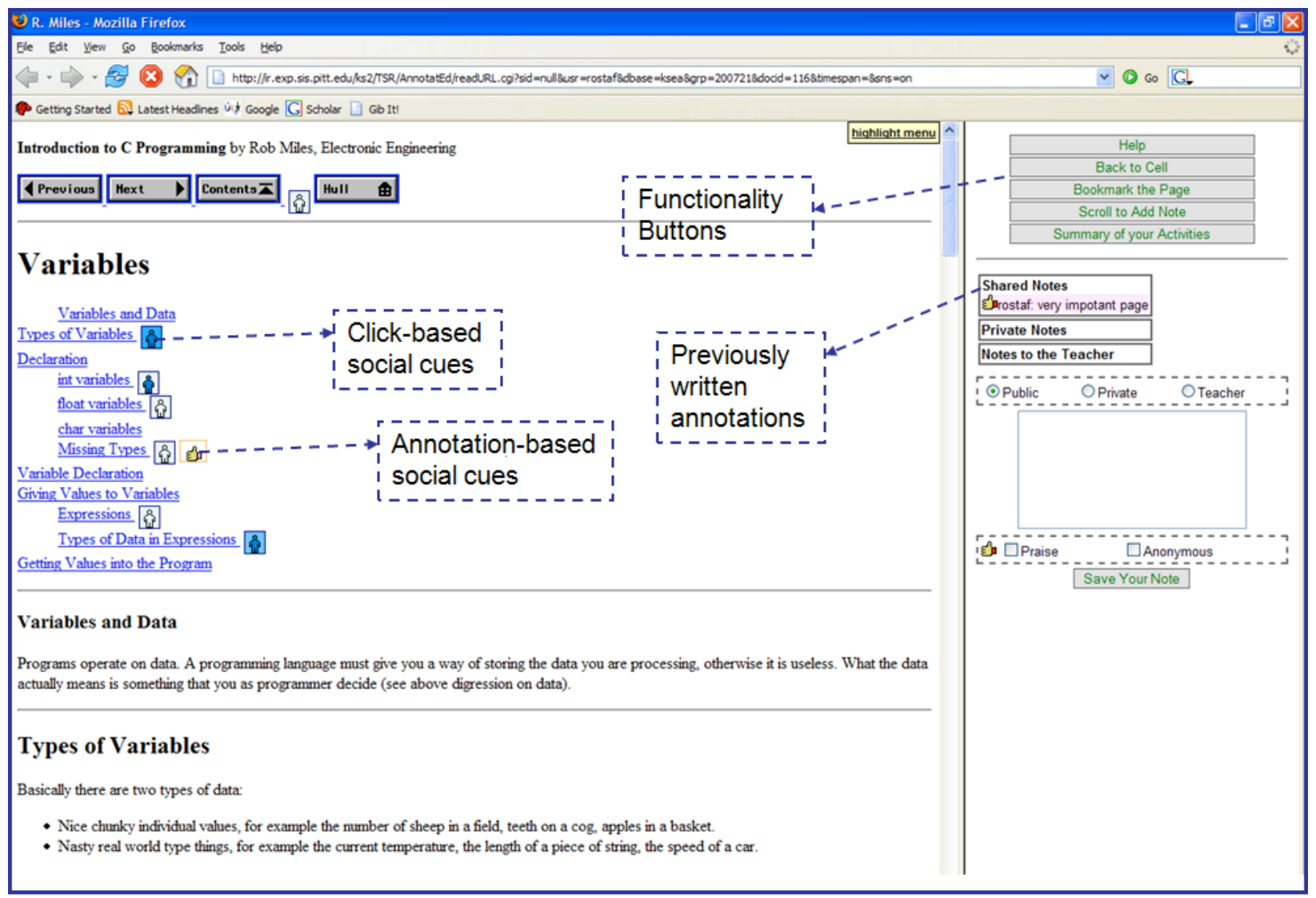

Figure 1. Sample page in AnnotatEd

\subsection{AnnotatEd and Knowledge Sea II}

Knowledge Sea II was designed to help students navigate from weekly lectures to relevant online educational materials by using a map-based information visualization and horizontal navigation between resources. AnnotatEd is an important component of Knowledge Sea II, which enables the system to provide traffic-based (Brusilovsky et al., 2004) and annotation-based (Farzan and Brusilovsky, 2005) social navigation support.

Figure 2 shows the general view of Knowledge Sea II. The left side of the figure shows a map of resources, with related resources being located closer to each other. When the user clicks on any of the map cells, they will see a list of resources inside that cell. When the user clicks on an item in the list, the page opens up inside AnnotatEd. We call this type of navigation to

\footnotetext{
${ }^{*}$ http://portal.acm.org/
} 
resource pages - map-based access. In addition, the user may access resources using traditional browsing, by using the table of contents or clicking on links to move from page to page.

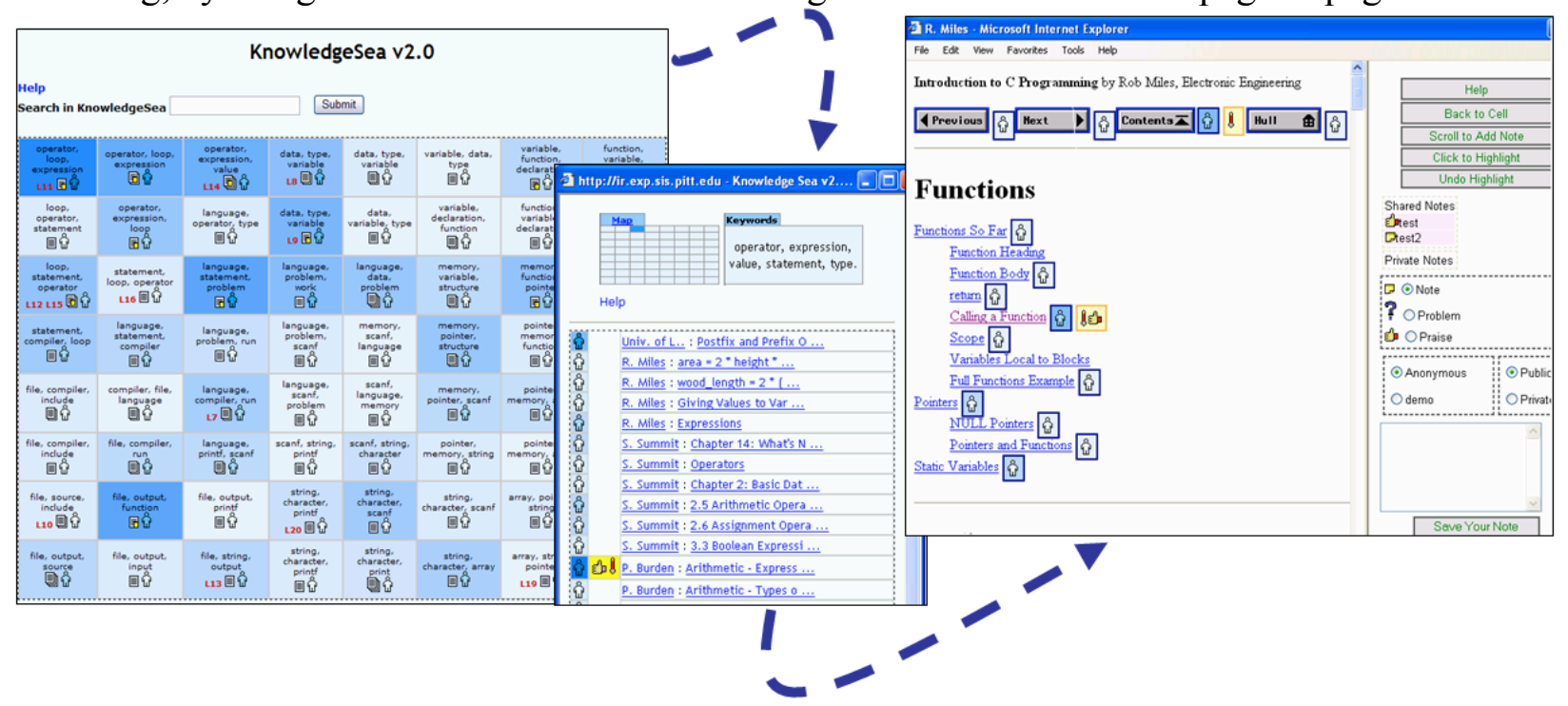

Figure 2. AnnotatEd in the context of the Knowledge Sea II System

All types of access are enhanced by the social navigation support. Each link inside the page shown by AnnotatEd is annotated with two different icons: the Traffic icon and the Annotation icon. These icons are visible on the right side of Figure 2 and are shown in more detail in Figure 3. The Traffic icon represents traffic-based SNS. The background color represents the volume of visiting activity completed by a group of users (in this case, a class). The higher the intensity of the background color, the higher the group activity for that page. The concept of visiting activity or traffic integrates the number of visits and the time spent visiting. The human figure represents the individual's visiting activity. The intensity of its color represents the volume of the individual's traffic. In a similar way, the Annotation icon represents the number of annotations on the page made by the user (a note is present and ranked by color) and the group (denoted by background color).

This map is not a part of AnnotatEd, but it displays the same traffic- and annotationbased social navigation support cues using information about user navigation and annotation that has been collected in AnnotatEd. The background color of each cell and the color of the human icon inside the cell represent, respectively, the cumulative traffic produced by the group and the user while visiting pages clustered in the cell. Moreover, the cells are augmented with user annotations. For each document inside the cell, information about interactions and annotations is collected by AnnotatEd and the accumulated data is visualized on the map. In addition, each link inside a map cell is also augmented with traffic- and annotation-based information that has been collected by AnnotatEd using the same approach as AnnotatEd uses to annotate links inside pages.

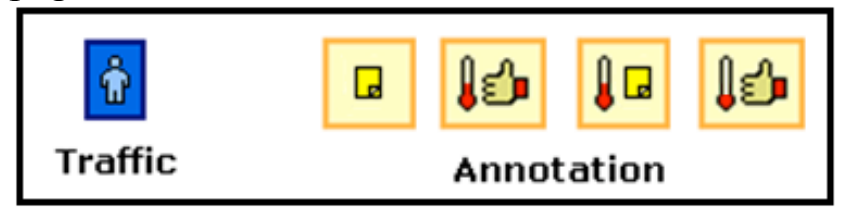

Figure 3. Navigational Cues 


\subsection{AnnotatEd and ASSIST-ACM}

Similar to its usage in Knowledge Sea II, AnnotatEd is used in the ASSIST-ACM system to help students locate papers that are relevant to their information-seeking goals (Freyne et al., 2007). AnnotatEd keeps track of all papers visited by any member of the group and allows the members to annotate papers as being relevant or irrelevant to the group. AnnotatEd uses this information to augment the links with visual cues. ASSIST-ACM shows that visual cues generated by AnnotatEd can be tuned to the specific characteristics of each host system and its interface. ASSIST-ACM uses different fill levels for the footprints icon (Figure 4) instead of background color, to represent group visiting activity. The fill levels represent different degrees of visiting activity, with higher levels representing higher traffic.

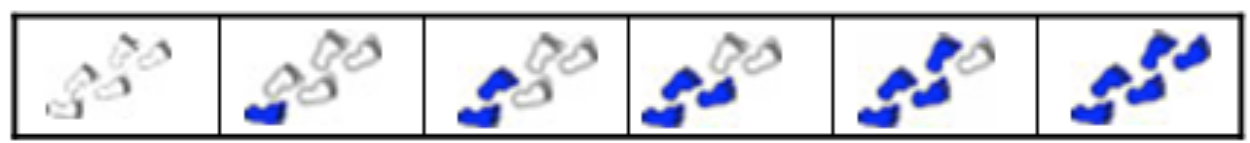

Figure 4. Visual icons representing the different magnitudes of group visiting activity

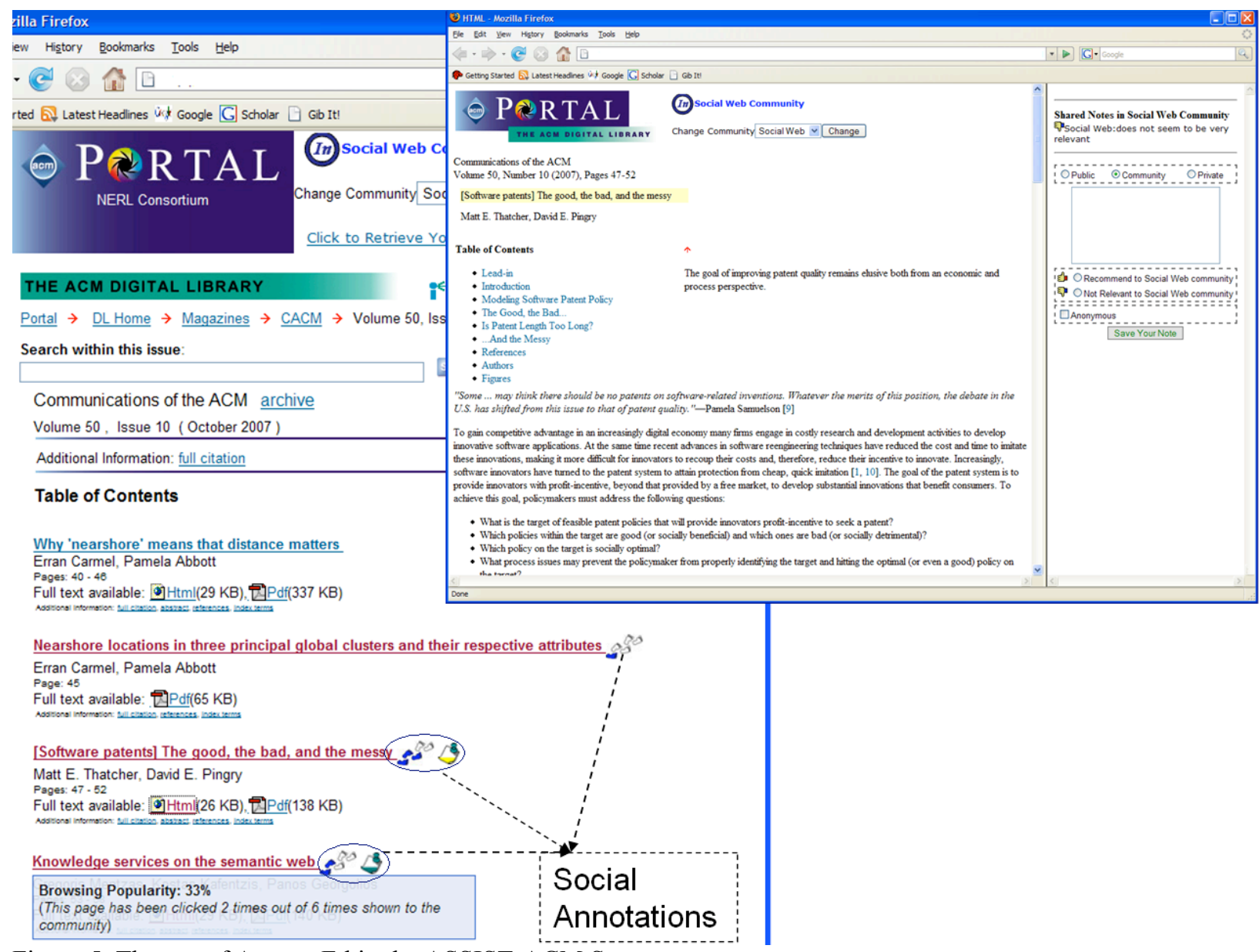

Figure 5. The use of AnnotatEd in the ASSIST-ACM System

Calculation of the color level can be adapted to the specific application as well. For example, in the ASSIST-ACM system it was important to take into account both positive and negative social evidence. In this context, the users look at a list of papers each time and decide 
whether to check a specific paper or not. Information about unvisited papers is as important as information about visited papers: i.e. unvisited papers are irrelevant to the group or user information-seeking goal. Therefore, we compute the fill level by taking into account the number of times a paper is visited over the total number of times that the paper is shown to the users. Figure 5 presents a sample page table of contents page in ASSIST-ACM, where the links are augmented with social cues and an article page is opened in AnnotatEd. The details about the usage of AnnotatEd in the ASSIST-ACM system can be found in (Farzan et al., 2007).

\section{Traffic-based SNS in AnnotatEd}

The implementation of traffic-based annotation support in AnnotatEd went through two stages. We started with a rather simple "footprint" mechanism for social navigation, previously discovered in earlier works (Wexelblat and Mayes, 1999). While this mechanism appeared to be quite helpful, user studies revealed some problems. On the positive side, we found that footprintbased navigation, with its "pragmatic" click-counting interpretation, can achieve success in helping users find their way through hyperspace. On the negative side, our experience and user feedback pointed out that click-based footprints can mislead users, due to lack of accuracy. The number of clicks is not a reliable evidence of quality and relevance for the visited page. Page selection is done on the basis of the link anchor alone. So poor quality or irrelevant pages may not be always avoided by looking at the anchor. A short visit to the page may be required to realize whether it is relevant or not. Log analysis data from the two semesters of classroom study of Knowledge Sea II shows that about $60 \%$ of page visits are relatively short. Pragmatic counting does not distinguish these unproductive visits and lengthier ones, so the simple footprint mechanism can mislead the students towards non-important pages. A comparison of the quality of often, but quickly-visited pages to pages with fewer but longer visits, shows that a significantly higher percentage of pages with long visits are noteworthy to the students. We define noteworthy pages as the ones which attract student annotations (Farzan and Brusilovsky, 2005). To resolve these problems, we enhanced this mechanism to take into account time spent reading. This section briefly introduces the original mechanisms and then presents the details of the new mechanism, which is now used in both the Knowledge Sea II and ASSIST-ACM systems presented above.

\subsection{Stage I: Counting Page Visits}

The first version of AnnotatEd implemented a "footprint" mechanism for social navigation (Wexelblat and Mayes, 1999). The mechanism tracked all page-visiting activities (known as traffic) and made it visible to the community of users through link annotation. Links inside the pages were annotated with visual cues indicating individual and group traffic. Trafficbased visual cues are a combination of a human icon on a colored square, where the background color represents the visiting activity of the group and the color of the human icon represents the individual's visiting activity. There are 10 levels of background color as shown in Figure 6.

\begin{tabular}{|l|l|l|l|l|l|l|l|l|l|l|}
\hline Click Level & 1 & 2 & 3 & 4 & 5 & 6 & 7 & 8 & 9 & 10 \\
\hline Color & & & & & & & & & & \\
& & & & & & & & & & \\
\hline
\end{tabular}


Figure 6. Background color levels representing the magnitude of group visiting activity

To help distinguishing the color difference between low and high traffic easily, the intensity of the color increases logarithmically. The traffic level is computed by tracking the users' page visits. The formula used for computation and a motivation for it can be found in (Brusilovsky et al., 2004).

\subsection{Stage II: Taking into Account the Time Spent Reading a Page}

To capture a more precise insight into the intention of the users and to eliminate potential "tar pits" (e.g., the user was attracted by a likely-relevant link and visited the document, but immediately backed away after discovering that it was not really good or relevant), we take into account time spent reading (TSR) each page. A "real visit" to a document (based on the TSR) is computed as shown in figure 7.

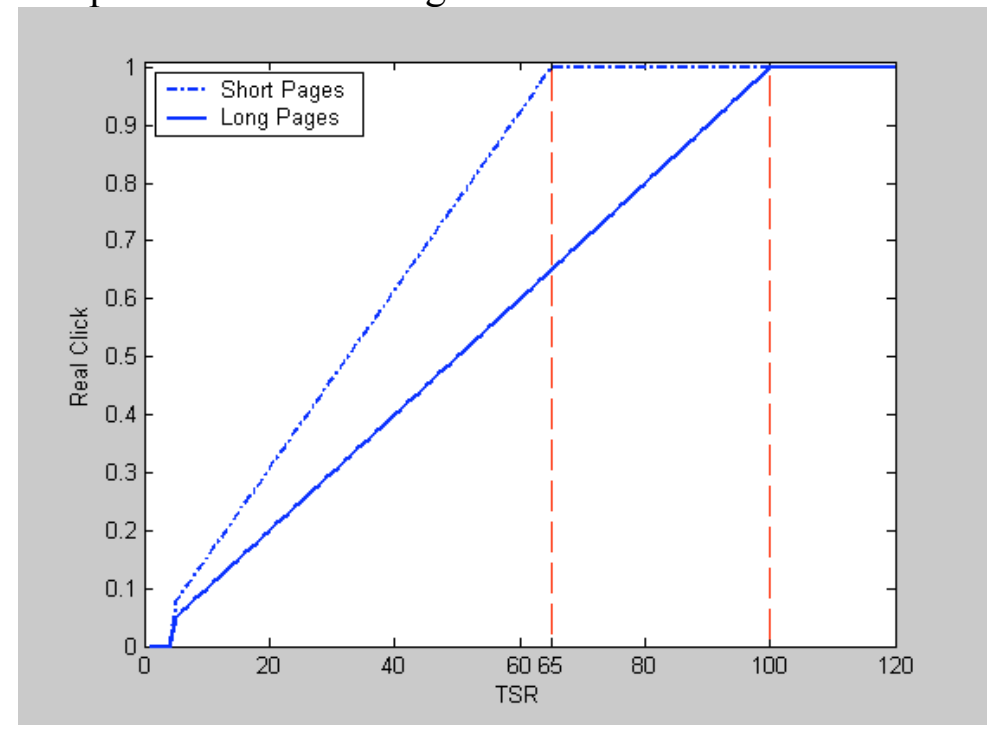

Figure 7. Computation of real visit based on TSR

If the TSR is too short (less than 5 seconds) or too long (more than 10 minutes), the page visit is ignored. We have defined two thresholds for long and short pages based on our collected data from observing students' interaction with the system. If the TSR is below this threshold, only a partial "click" is credited as a proportion computed by dividing the TSR by the threshold. Only if the TSR is more than the threshold is the click counted as one real visit. formula:

Once the real visit is computed, we compute the traffic level by using the following

$$
\text { ClickLevel }=\left\lfloor\left(\frac{\log (\text { Click })-\log (\text { Min })}{\log (\text { Max })-\log (\text { Min })}\right) * \text { noOfIntervals }\right\rfloor
$$

Where Min is the minimum possible number of group visits for a page, which we set to zero; while Max is maximum possible number of visits to a page, which is dependent on the number of students in the group, while the noOfIntervals is the possible number of different colors. To make the color changes great enough that small changes in a relatively low number of clicks will be immediately reflected in color changes and noticed by the user, we used a 
logarithmic transformation. Finally, normalization is performed to get the color level. Figure 8 shows an example where group and individual traffic levels aren’t proportional.

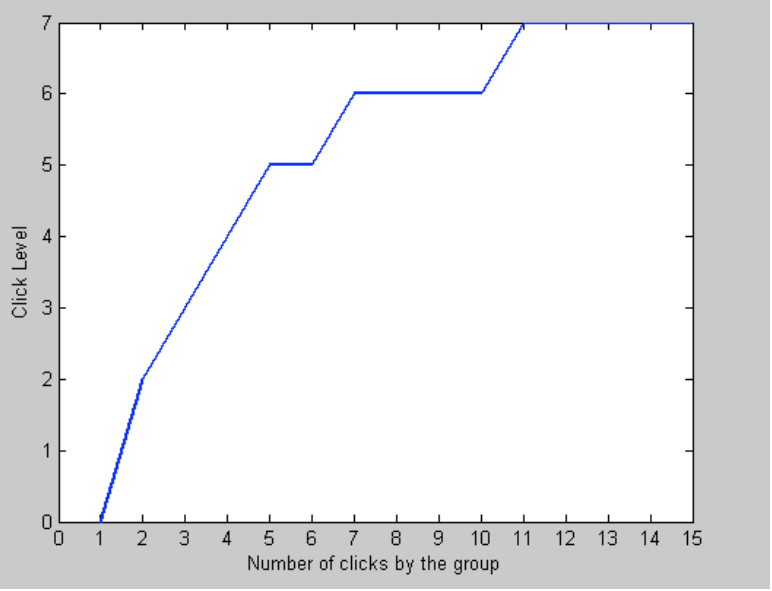

Figure 8. Example of traffic levels, according to number of clicks

The color of the human figure in the Traffic icon (Figure 2) shows the magnitude of individual visits, which is represented with 5 color intensity levels. The color intensity is calculated the same way as explained above. Figure 9 shows five human icons ranging from a dark blue to a very light blue color.

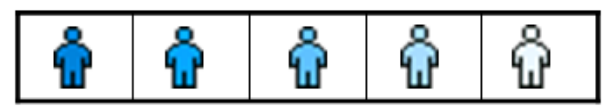

Figure 9. Visual cues representing different levels of individual visiting activity

\section{Annotation-based SNS in AnnotatEd}

The current version of annotation-based SNS, used in Knowledge Sea II and ASSIST ACM, was developed over three design cycles. Each cycle was followed by extensive user studies. We learned a number of lessons during these studies and we think these lessons may be important to the reader. Instead of simply describing the current implementation of annotationbased SNS, this section begins by presenting the two earlier versions along with lessons learned and the rationale which guided our design decisions.

\subsection{Stage I: A Simple Annotation Interface}

AnnotatEd began with a very simple annotation interface accompanied by a complete tracking feature. The first version provided an easy-to-use interface for annotating educational resources by letting students add comments to the page. Students were not able to share notes with others and were not aware of annotations by others.

Our preliminary evaluation of annotations showed that about $60 \%$ of the students appreciated the ability to annotate and further, were interested in sharing their annotations, seeing annotations made by others, and knowing which pages were annotated. In addition, we examined notes written by the students and discovered that the notes could be categorized into three groups: praise (a positive point about the page), problem (a negative point about the page), or general (a neutral statement, such as, "I have to go back to this page"). Considering this 
classification, 37 notes were classified as "praise," 36 as "problem," and 34 as "general." This data motivated us to proceed with expanding the user's ability to annotate and also to explore the use of annotation as the source of a more reliable SNS.

\subsection{Stage II: Introduction of Annotation-Based Navigation Support}

In the second stage of the implementation of AnnotatEd, the students were able to view public notes written by others. We added the ability to make public and private annotations and to choose one of the above three categories for annotations (praise, problem, or general note). We also allowed students to choose to sign notes or to keep them anonymous. This was all added in order to motivate students to share feedback with their classmates. Figure 10 presents the annotation interface of AnnotatEd, at that stage. The top part of the annotation interface displays previously written notes.

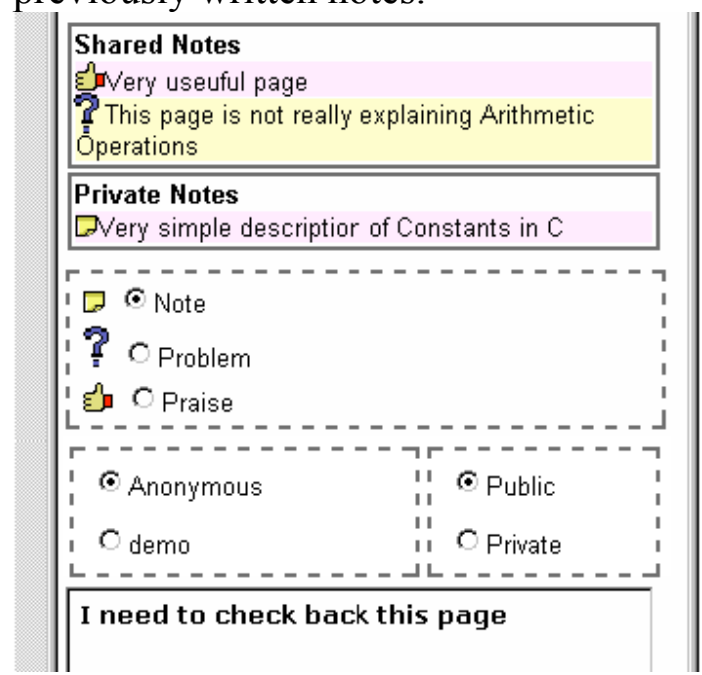

Figure 10. The Annotation Interface (Stage II)

At this stage, we added basic annotation-based SNS by augmenting links to pages with public annotations with the Annotation icon. Similar to the Traffic icon, the background color of the Annotation icon represents the magnitude of group annotation activity and the color of the sticky note icon represents the magnitude of individual annotation activity. Here the background has 3 levels and the foreground has two levels. Since the number of annotations is not very large for each given paper, we used a fixed threshold for different color levels, as shown in Figure 11.

\begin{tabular}{|l|l|l|l|}
\hline Annotation Level & $<=2$ & $2<<=4$ & $>4$ \\
\hline Color & & & \\
\hline
\end{tabular}

Figure 11. Background color levels representing the magnitude of group annotation activity

The evaluation of the second stage made us aware of some interface problems. After an analysis of student annotations we discovered that the difference between types of annotations was not very clear to the students. First of all, many obviously positive annotations were categorized by the students as being merely "general" notes: $61 \%$ of the annotations that were rated as "general" were really "praise." We hypothesized that the interface may have triggered this problem. As shown in Figure 10, in order to write a note, students must first choose the type, then the privacy level and authorship, then write the note, and click to save the note. The default type is set to general and changing the type from default to any other case is optional. 
We think that the box for selection of type was too distant from the saving note button, so that the students could easily forget to change the type, when done. Secondly, "problem" annotations were used not to indicate a negative page (as we had assumed), but to report problems with the page to the teacher.

\subsection{Stage III: Enhanced Annotation and Navigation Support}

To address interface issues encountered in Stage II, we removed the "problem" type and added the option to write a note that could only be seen by the instructor of the course. The note to the instructor could serve as a way to report problems within the educational material. We also rearranged the interface and placed important options to consider closer to the "save note" button (Figure 12). Following the results of our study of the use of signed and anonymous notes (see section 7.2), the signed option became the new default option.

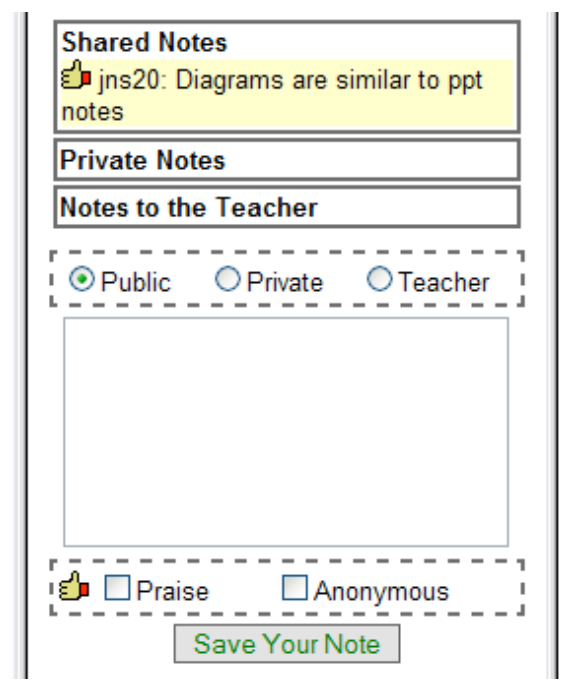

Figure 12. The Annotation Interface (Stage III)

In the third stage we also enhanced both the annotation and navigation sides of the system. To allow for a simpler form of annotation, we added highlighting. To highlight, one merely selects any part of the text and clicks on the "highlight" button. Highlighting creates an effect similar to that of using a highlighter on paper.

We also enhanced annotation-based navigation support by visualizing annotation temperature and by using the annotation type which had been provided by the student author. The Annotation icon was modified to represent this information. The background color of the square represented the same information about the density of group annotations, but the sticky note icon inside the square was replaced by a thumbs-up icon to indicate a positive annotation from the logged-on user. We used the sticky note to indicate the existence of a general note. Similar to the previous stage, the color of the foreground icons represented the density of individual notes. In addition, a thermometer icon was added to present the "overall temperature" of the annotations made by the group of students. The temperature grew warmer when a page attracted more positive annotations and colder when it attracted more problem-type annotations (Figures 2 and 3). 


\section{Evaluation}

We focus this evaluation section on the application of AnnotatEd to the Knowledge Sea II system. Knowledge Sea II has been used in the School of Information Sciences at the University of Pittsburgh for over six semesters and has been evaluated at the end of each semester. We are reporting the results obtained during the evaluation of three versions of AnnotatEd during the first four semesters it was used. The studies were done in an introductory $\mathrm{C}$ programming course (undergraduate), and an information retrieval course (graduate). Table 1 presents general statistics about the number of students in the classroom for each study and the number of students who used the system. It also shows the number of unique documents being accessed by the students and the maximum number of visits to a document.

\begin{tabular}{|r|l|l|l|l|l|l|}
\multicolumn{7}{|c|}{ Table 1 - General usage statistics } \\
\hline & Course & $\begin{array}{l}\text { System } \\
\text { Stage } \\
\text { Students }\end{array}$ & $\begin{array}{l}\text { Total } \\
\text { students } \\
\text { using } \\
\text { system }\end{array} \quad$ the & $\begin{array}{l}\text { Unique } \\
\text { documents } \\
\text { accessed }\end{array}$ & $\begin{array}{l}\text { Maximum } \\
\text { visits to a } \\
\text { document }\end{array}$ \\
\hline Fall 2003 & C & I & 27 & 18 & 284 & 20 \\
\hline Spring 2004 & C & II & 26 & 25 & 431 & 18 \\
\hline Fall 2004 & C & III & 15 & 12 & 368 & 22 \\
\hline Fall 2005 & IR & III & 15 & 13 & 153 & 19 \\
\hline
\end{tabular}

In each class, the system was introduced by the instructor. The students were able to access the system anytime, anywhere. During the study, all the students' interactions with the system were logged for log analysis. We also administered a questionnaire at the end of each semester, to collect the students' subjective opinions. Following the developmental stages of the system, we ended each semester by evaluating the main new features of that particular system. The following subsections describe the evaluation goals of each stage.

\subsection{Stage I}

As described above in the first stage, while students had the ability to write annotations, no annotation-based social navigation was supported and students were only able to view their own annotations. A subjective evaluation was designed to discover the students' opinion about the ability to annotate and their desire to share their annotations with others, know which pages had been annotated by others, and see the content of others' annotations. The questions are shown in Figure 13. The first question was designed to assess the overall value of the annotation feature and the other two questions were designed to assess the potential value of adding social navigation support. 


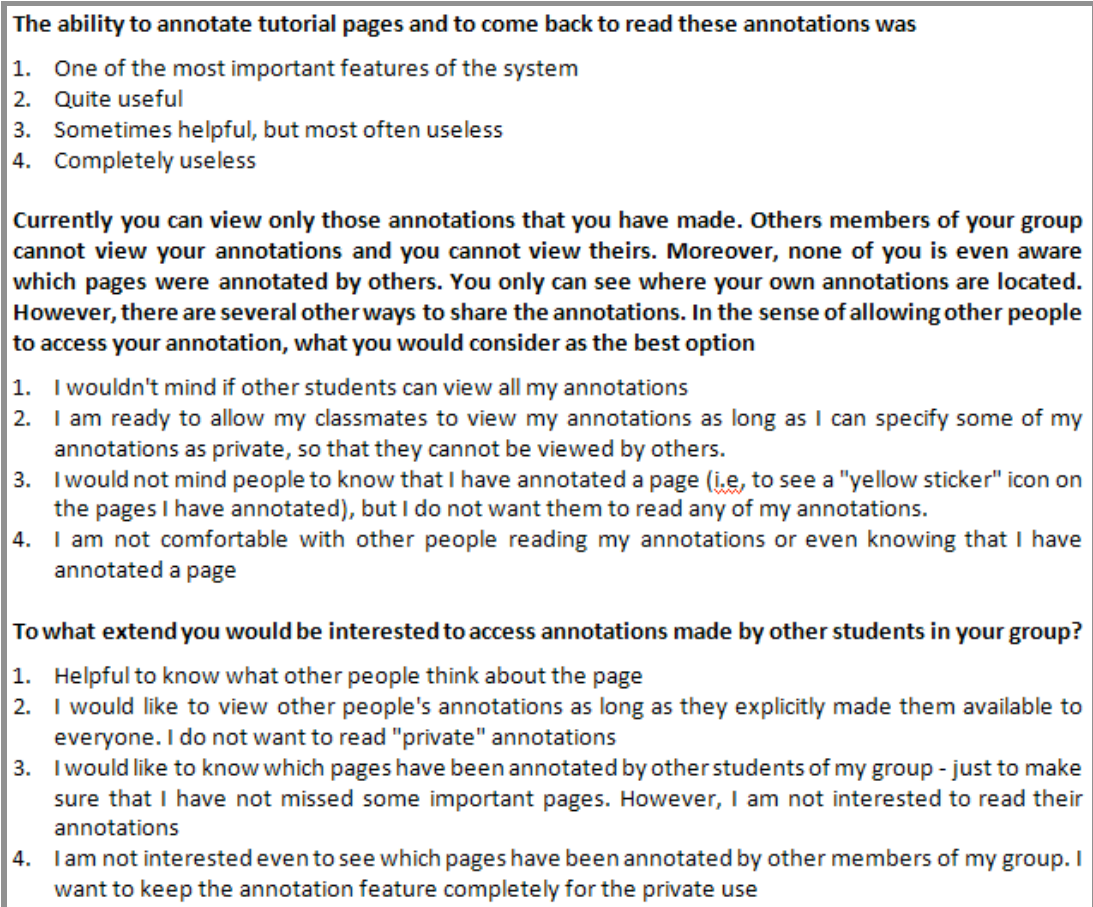

3. I would not mind people to know that I have annotated a page (i.e, to see a "yellow sticker" icon on the pages I have annotated), but I do not want them to read any of my annotations.

4. I am not comfortable with other people reading my annotations or even knowing that I have annotated a page

To what extend you would be interested to access annotations made by other students in your group?

1. Helpful to know what other people think about the page

2. I would like to view other people's annotations as long as they explicitly made them available to everyone. I do not want to read "private" annotations

3. I would like to know which pages have been annotated by other students of my group - just to make sure that I have not missed some important pages. However, I am not interested to read their annotations

4. I am not interested even to see which pages have been annotated by other members of my group. I want to keep the annotation feature completely for the private use

Figure 13. Stage I: Subjective questions evaluating annotation features

The result is shown in Figure 14. The first column (Q1) shows that more than $60 \%$ of the students found the annotation ability very important or quite useful and less than $10 \%$ found it useless. The second column (Q2) shows that $70 \%$ of students were willing to share all their annotations or specific ones with other students and there were only $30 \%$ who didn't want to share any information about their annotations with other students. The third column shows that $60 \%$ of the students want to know about annotations made by others as long as the authors are willing to share them. Overall the subjective opinion is very positive towards annotations and potential annotation-based social navigation support.

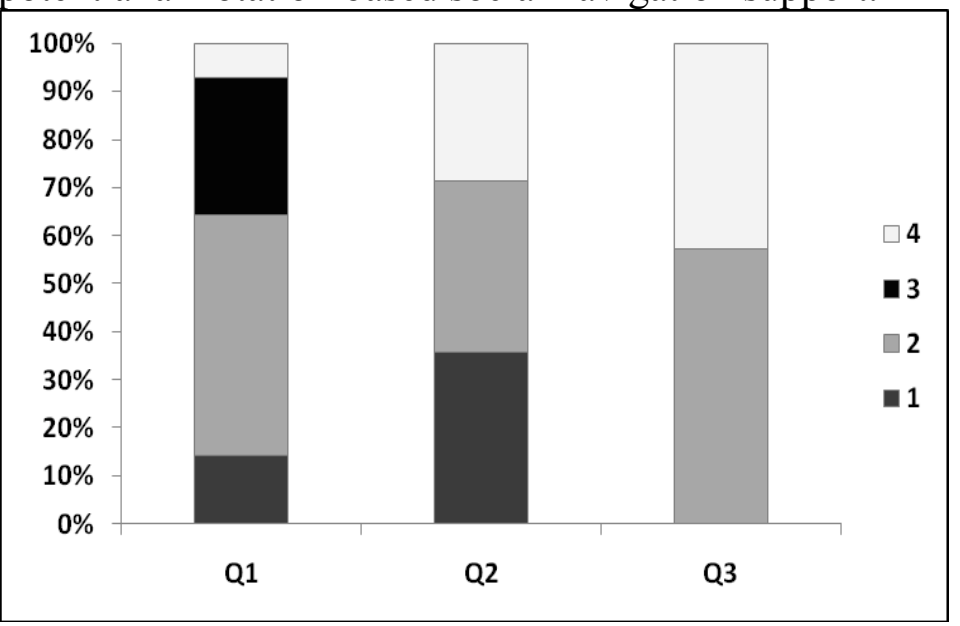

Figure 14. Stage I - Subjective opinions of students about the annotation features 


\subsection{Stage II}

The stage II evaluation was mostly formative. In that stage, annotation-based social navigation support was added to AnnotatEd and Knowledge Sea II. Therefore, the focus of our stage II evaluation was the value and effect of this additional functionality. We were interested in evaluating the effect of annotation-based social navigation support on the students' navigation behavior. We also were interested in assessing the value of some specific interface choices, such as the freedom to sign notes or to remain anonymous. Finally, we were interested in hearing the student's subjective opinions about the new features.

\subsubsection{The effect of Social Navigation Support on Link-Following}

To assess the influence of annotation-based visual cues on user navigation, we looked at the following:

1. The effect of annotation when the author revisited a page: We compared the chance of revisiting a page if the student had annotated it versus the chance of revisiting it if the page had not been annotated by the student. The results show that the chance of revisiting is $48 \%$ when the page is self-annotated and $17 \%$ when it is not. The difference is statistically significant ( $\mathrm{p}$-value $=0.01$ )

2. The effect of annotation on group navigation behavior: We computed the normalized access rate before and after the presence of public annotations. To normalize, we divided the number of page-visits by the number of possible days to access a page. Namely, the number of visits before annotation was divided by the number of days between the system's first use and the date of its first public annotation, while activity after annotation was divided by the number of days public annotation had been used in the system. Figure 15 shows that the normalized access after public annotations were introduced is significantly larger than before the availability of public annotations ( $\mathrm{p}$-value $<0.001)$.

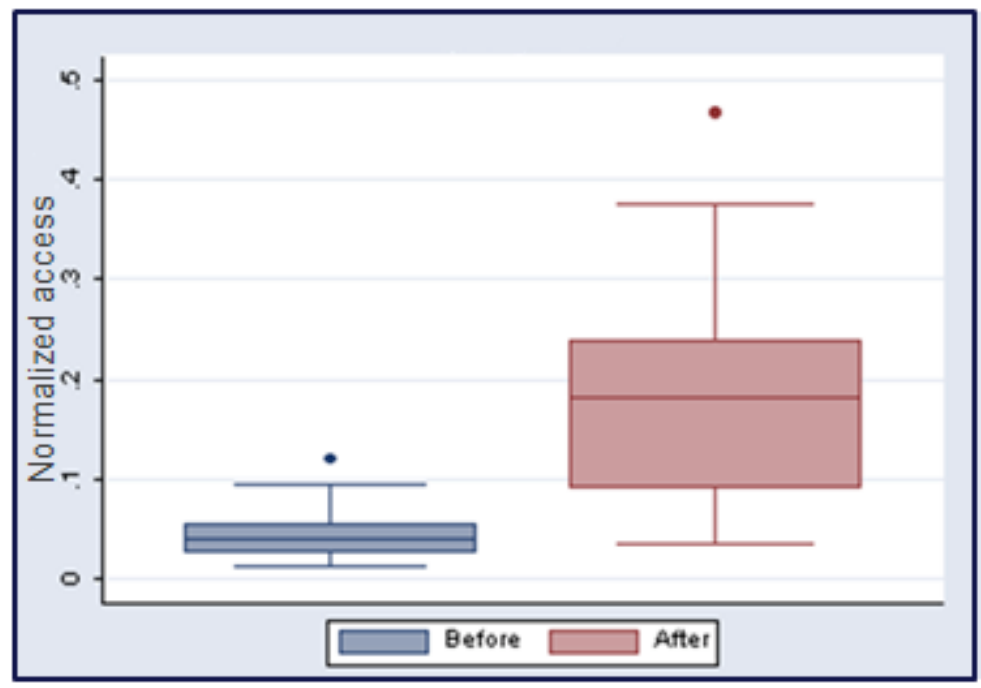

Figure 15. Effect of the presence of public annotation on student visits 


\subsubsection{The value of signed notes}

To assess the value of choice between anonymity and signed annotation provided in the Stage II interface, we compared the percentage of anonymous annotations with annotations signed by the user with the username. As can be seen in Table 2, students preferred to sign their annotations in the majority of cases. The result is rather notable considering that the anonymous annotation was the default option and thus students have consciously chosen to sign their annotations (Figure 10). The difference is statistically significant (prtest - test on the equality of proportions, $\alpha=0.05$ )

\begin{tabular}{|c|c|c|} 
Table 2 - Quantity of anonymous versus signed \\
annotations
\end{tabular}

Moreover, we were interested in comparing the quality of annotations written in the anonymous and signed formats. We hypothesized that when students sign their annotations they are more likely to write higher quality annotation, since it affects their credibility among their peers. To measure the quality of the annotations, we considered the type and length of the annotations: If the annotation had a type (problem or praise) it included more information and thus was considered more desirable. Also we assumed that longer annotations included more information; although this is not a straightforward assumption and needs to be evaluated more closely. To evaluate our hypotheses, we looked at the percentage of typed annotation in anonymous and signed formats. As shown in Table 3,67\% of anonymous annotations were in the general format while only $40.6 \%$ of the signed annotations were general.

\begin{tabular}{c} 
Table 3 - Type of anonymous versus signed \\
annotations \\
\hline \\
\hline
\end{tabular}

We also compared the length of anonymous versus signed annotations and, as shown in Figure 16 , signed annotations have a higher length on average and the difference is statistically significant (ttest, $\alpha=0.1$ ). The result supports our hypothesis that students produce higher quality annotations in the signed format. 


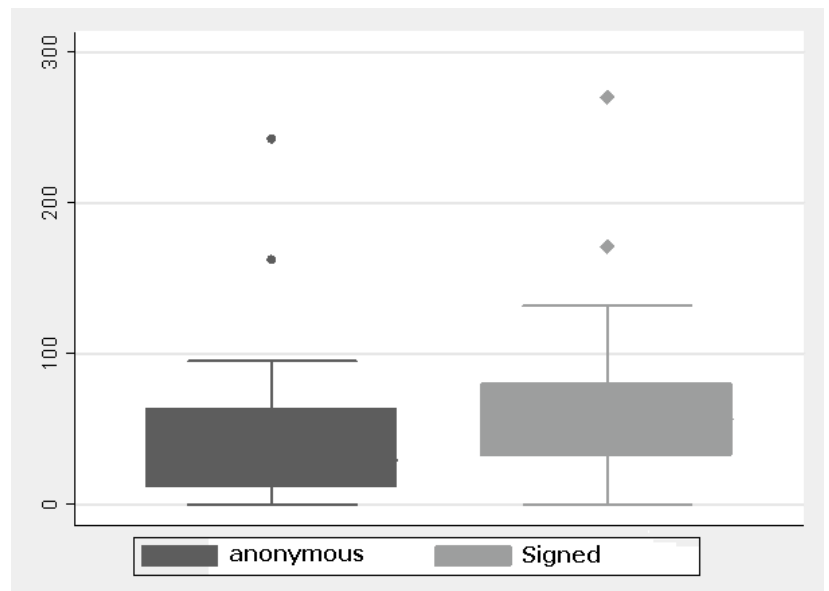

Figure 16. The length of anonymous versus signed annotations

\title{
7.2.3 Subjective evaluation
}

\author{
The ability to annotate tutorial pages and to come back to read these annotations was \\ 1. One of the most important features of the system \\ 2. Quite useful \\ 3. Sometimes helpful, but most often useless \\ 4. Completely useless
}

In current system you could choose one out of 3 types (e.g. Praise, Problem, General) when writing new annotations. In this sense, what you would consider as the best option

1. Typed annotations are helpful and it is good to have more than three specific types for annotations

2. Typed annotations are helpful and three existing types for annotations is just right

3. Typed annotations are confusing, best option is to have just one general type for annotations

To what extend you are interested to access annotations made by other students in your group?

1. I would like to view other people's annotations, it is helpful to know what other people think about the page

2. I would like to view other people's annotations as long as they explicitly made them available to everyone. I do not want to read "private" annotations

3. I would like to know which pages have been annotated by other students of my group-just to make sure that I have not missed some important pages. However, I am not interested to read their annotations

4. I am not interested even to see which pages have been annotated by other members of my group. I want to keep the annotation feature completely for the private use

The "density" of public annotations for a tutorial page is currently shown using several shades of yellow next to the link to this page. To what extent you are interested to know the "density" of public annotations when selecting best link to follow?

1. Very interested

2. Quite interested

3. Does not matter

4. Showing density of annotations makes no sense and only makes the interface confusing.

To what extent you would be interested to have the ability of highlighting or underlining the content of tutorial pages (similar to what you can do with highlighter when you are reading a paper based material)

1. Very interested

2. Quite interested

3. Does not matter

4. Not interested at all

Figure 17. Stage II - Subjective question evaluating annotation features and annotation-based social navigation support 
Stage II questionnaire was designed to assess the students' opinions about the value of annotation-based social navigation support in addition to the ability to annotate in general. The key questions are shown in Figure 17.

The results of the subjective evaluation is shown in Figure 18. Q1 shows that more than 50\% of the students found the ability to annotate very important or quite useful and less than $10 \%$ found it useless; but about $40 \%$ of the students found the ability to annotate only sometimes helpful. Q2 shows that $82 \%$ of students liked to specify a type while writing a note. Q3 shows that $80 \%$ of the students were willing to share all of their annotations or specific ones with other students and there were only $10 \%$ who didn't want to share any information about their annotations with other students. Q4 shows that the density of public annotation mattered to more than $70 \%$ of the students. Q5 assesses the interest of the students in how they viewed the ability to highlight, in addition to writing notes. The results show that the ability to highlight was appealing to more than $70 \%$ of the students. Overall, the students' subjective opinions were very positive towards annotations and students were in favour of annotation-based social navigation support.

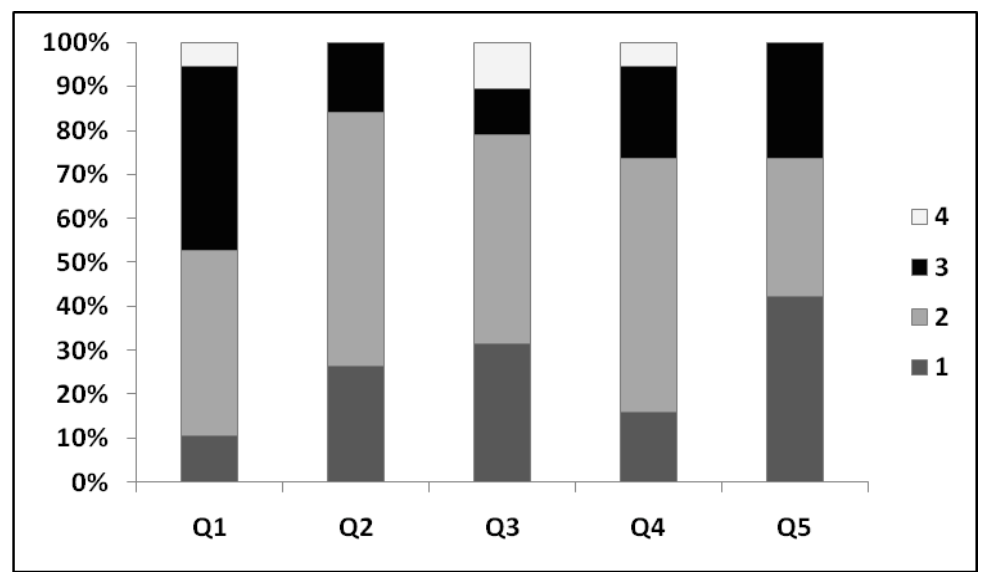

Figure 18. Stage II - Subjective opinions of students about the annotation features

\subsection{Stage III}

The third stage included the most recent annotation interface and annotation-based social navigation support as described in the paper. As a result, the evaluation was more deep and summative, by nature. The study included the evaluation of the system over two semesters in an undergraduate $\mathrm{C}$ programming course and a graduate Information Retrieval course. In this stage, we were interested in evaluating the following hypotheses:

1. Providing navigation support inside Web pages (browsing) would be important, because browsing-based access to content is vital for the students. We expected to observe a considerable usage of browsing, even with the presence of other access options such as search and map-based navigation.

2. Annotating links with SNS cues would affect the students' decision of which link to follow.

3. Pages with annotation-based SNS are important pages.

4. Students would appreciate the annotation functionality of the system.

5. Students would appreciate the annotation-based SNS inside Web pages. 


\subsubsection{Browsing versus Map-based Navigation and Search Access}

As mentioned before, AnnotatEd is connected to Knowledge Sea's navigation map. The whole system also offers a search interface. Therefore, the same educational materials can be accessed in different ways. The same navigation support is provided for all three access methods (map, search, browsing). To evaluate our first hypothesis, we compared the usage of these three accessing methods by tracking the source of each resource page access. Although map-based navigation is known to be advantageous, the overall number of accesses through browsing was higher than the number of map-based accesses (Figure 19). Also, while searching is considered to be the most important information access method on the Web, our results show that actually the lowest number of accesses was done through searching.

It is worth noticing that the usage of search in the information retrieval course was significantly higher than in the $\mathrm{C}$ programming course. This may be related to the fact that graduate students, especially those taking an information retrieval course were much more skilful and confident in using searches. However, the data also shows that independent of the level of students' expertise, browsing was important. Over both semesters, about $50 \%$ of the accesses were done through browsing. The results support our first hypothesis and agree with the expected importance of browsing as a method of accessing information on the Web.

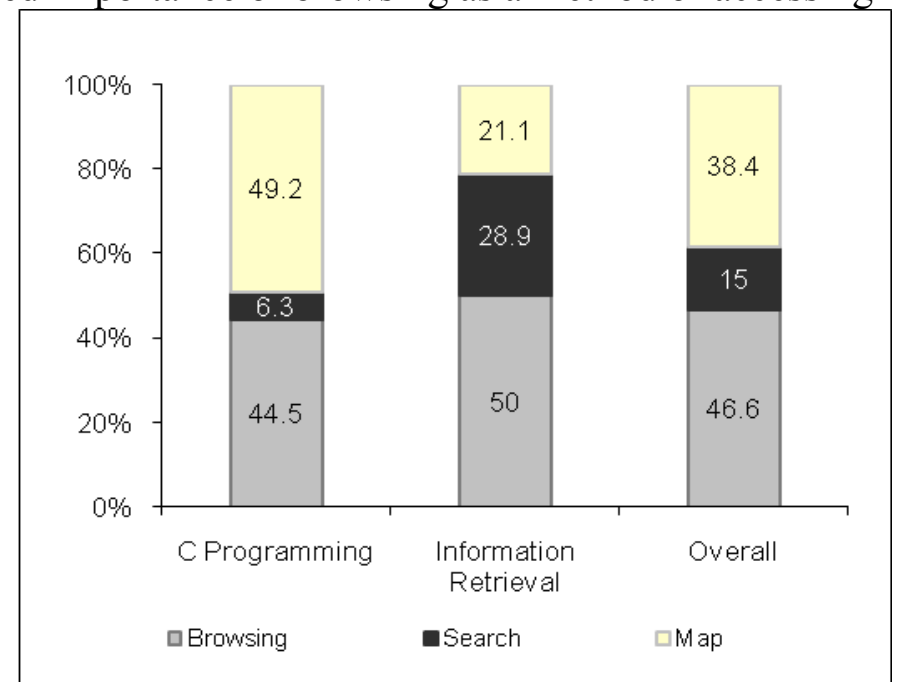

Figure 19 - Percentage of access from each method

\subsubsection{Browsing versus Map-based Navigation: Helping the User to Find Relevant Pages}

Browsing seems to be very important for the students and they use it a lot. But does browsing help them to access pages they are looking for? We hypothesized that pages selected through browsing would be more relevant to the students, since they have more context from which to choose the page and thus should be more certain about the content of the page. To assess our hypothesis, we looked at the exact pages accessed from two different accessing methods: map and browsing. We found 62 pages where the same page had been accessed by both map-navigation and browsing. We measured the desirability of the page by the time the student spent on the page, since time spent has been proven to show a strong correlation to interest (Claypool et al., 2002).

Table 4 - Time spent on pages accessed through the map compared to time spent 


\begin{tabular}{|c|c|c|}
\hline \multicolumn{3}{|c|}{ when browsing (in seconds) } \\
\hline Browsing & Map & p-value (paired $t$ test) \\
\hline 104.84 & 64.24 & 0.028 \\
\hline
\end{tabular}

Table 4 presents the average time spent on a page accessed through the map compared with average time spent on the same page when accessed by browsing. We observed that students spent significantly more time on a page when they accessed it through browsing. This is a good indication that browsing provides the most reliable mechanism for the students to discover relevant and interesting pages. Together with the discovered fact that browsing is also a heavily-used information access approach, this fact allows us to argue that the presence of browsing is critical for educational information access systems. The importance of browsing also justifies the research on improving browsing-based access to educational resources such as the social navigation support approach explored in this section.

\subsubsection{The Effect of Social Navigation Support on Link-Following}

To evaluate the effect of annotating links with SNS cues, we compared the number of accesses to links with and without traffic-based or annotation-based SNS cues. As can be seen in Table 5, pages with traffic- or annotation-based SNS attracted a higher number of visits, with annotation-based SNS pages attracting the most visits. In both cases, the difference is significant $(\alpha=0.05)$. The results support our second hypothesis and suggest that annotation can play an important role in providing navigation support to users.

\begin{tabular}{|c|c|c|c|}
\hline \multicolumn{4}{|c|}{ Table 5 - Average number of clicks for pages with and without traffic/annotation-based SNS } \\
\hline & None & Traffic & Annotation \\
\hline Average number of clicks & 1.4 & 1.8 & 3.6 \\
\hline
\end{tabular}

Additionally, similar to stage II we computed the normalized access rate before and after the presence of public annotations. Namely, the number of visits before annotation was divided by the number of days between the system's first use and the date of its first public annotation, while activity after annotation was divided by the number of days public annotation had been used in the system. Figure 20 shows that normalized access after public annotations were created is significantly larger than before the availability of public annotations ( $\mathrm{p}$-value $<0.001$ ).

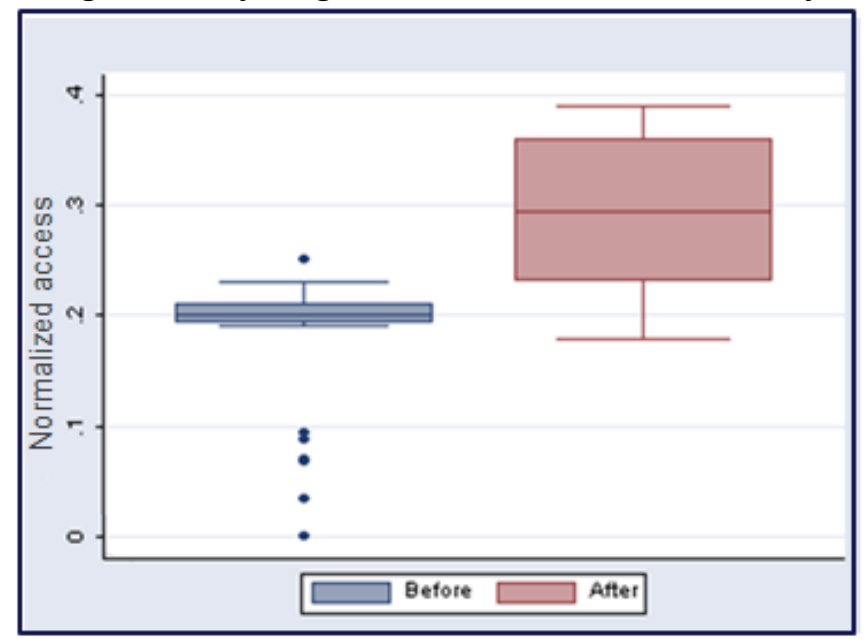

Figure 20. Effect of the presence of public annotation on student visits 


\subsubsection{Importance of Pages with Student Annotation}

Our results show that students are more likely to follow a link to a page that has annotations on it; therefore, it is essential to know whether this guidance is accurate, by assessing the quality of these pages. To evaluate the quality of annotated pages, we calculated the average access to the page before the page was annotated, in order to subtract out the effect of student annotation. We compared this to the average accesses to pages with no annotation. In both semesters, as shown in Table 6, pages with student annotations attracted significantly more clicks, even before they were annotated. This result suggests that pages, which eventually get annotated, are somehow important for the group. It supports our third hypothesis: Annotationbased SNS does lead the students to important pages.

Table 6 - Average access to pages with and without annotation, before the occurrence of student annotation

\begin{tabular}{|c|c|c|c|}
\hline & Annotated & Not Annotated & $\mathrm{p}$-value $(t$ test $)$ \\
\hline C programming & 3.15 & 2.02 & 0.03 \\
\hline Information Retrieval & 2.58 & 1.42 & 0.0005 \\
\hline
\end{tabular}

Note, this result does not contradict the finding in section 7.3.2 that annotations attract users' attention. As presented in section 7.2.1 and 7.3.3, the data shows that normalized access to annotated pages is significantly higher after being annotated (figure 15 and 20).

\subsubsection{Subjective Data Analysis}

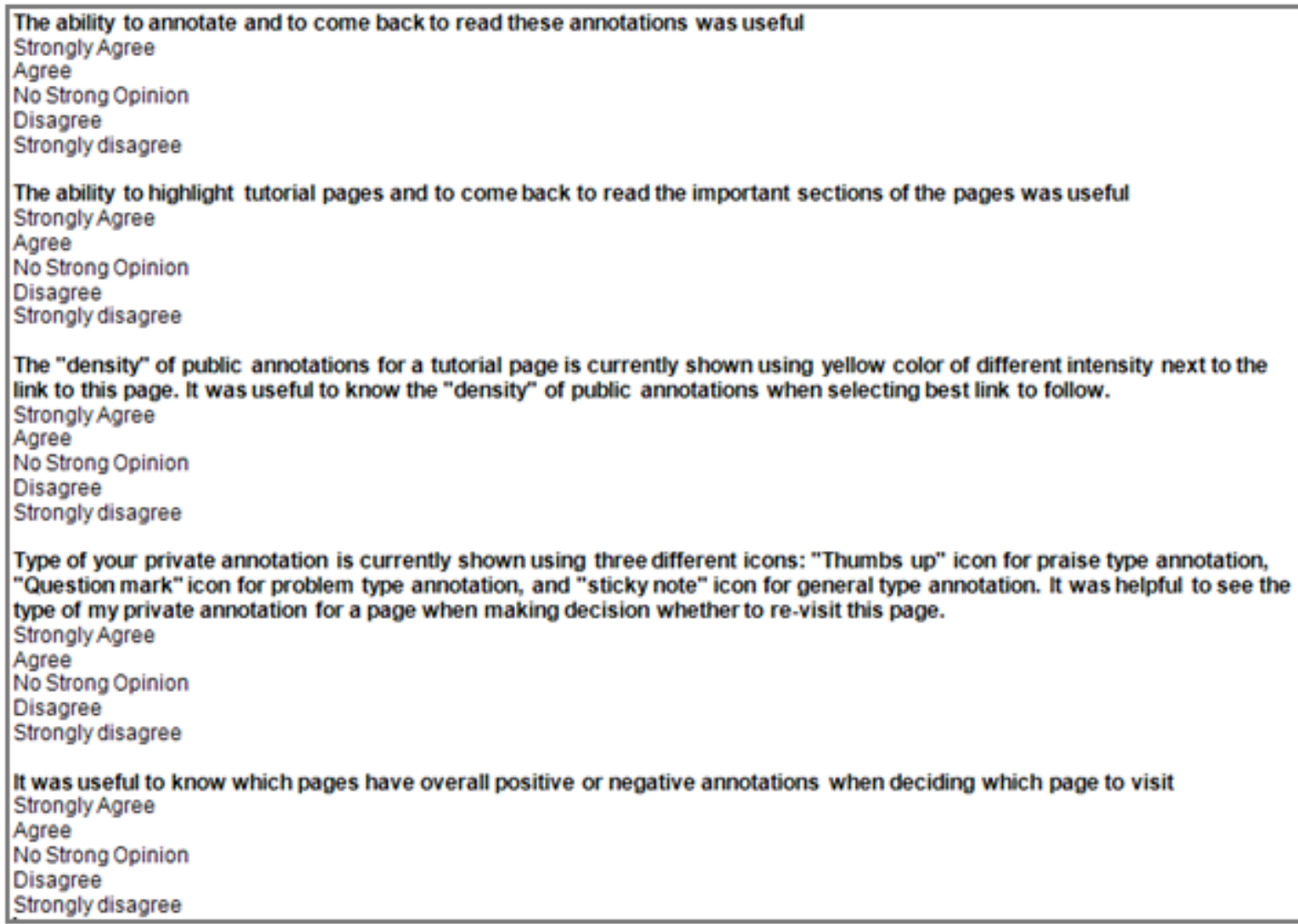

Figure 21. Stage III - Subjective question evaluating annotation features and annotation-based social navigation support 
Similar to previous stages, we conducted a questionnaire at the end of the semester to collect students' subjective feedback about different features of the system. Figure 21 shows questions related to annotation and annotation-based SNS. In the C programming course we asked separate questions about the note-writing and highlighting functionalities, while in the information retrieval course we asked one general question about annotation functionality, in order to decrease the number of questions asked. As shown in figure 22 , more than $80 \%$ of the students, in all cases, appreciated the ability to annotate as provided by the system. This data supports our 4 th hypothesis.

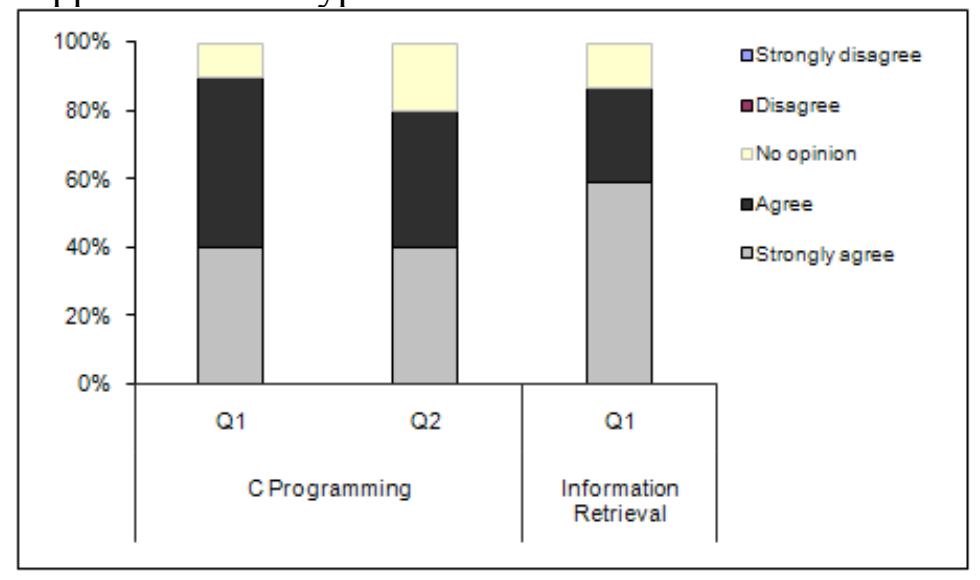

Figure 22. Student attitude towards the annotation functionality of the system

To evaluate student opinion about annotation-based SNS in a more detailed way, we asked 1) about the usefulness of displaying information about density of public annotations as a visual cue, 2) whether the private annotations offered by the system are important, and 3) whether the overall temperature of a link influenced them to follow a link. The questions were multiple-choice, asking the level of agreement with the statement in the question. The questions were exactly the same for both courses. As presented in Figure 23, the dominant majority of the students positively evaluated the main features of annotation-based SNS. Among the listed three features, the visualization of the density of public annotation appeared to be less successful, especially in the context of an undergraduate class. Yet even in this class, the feature was positively evaluated by about $70 \%$ of students. The result supports our 5 th hypothesis.

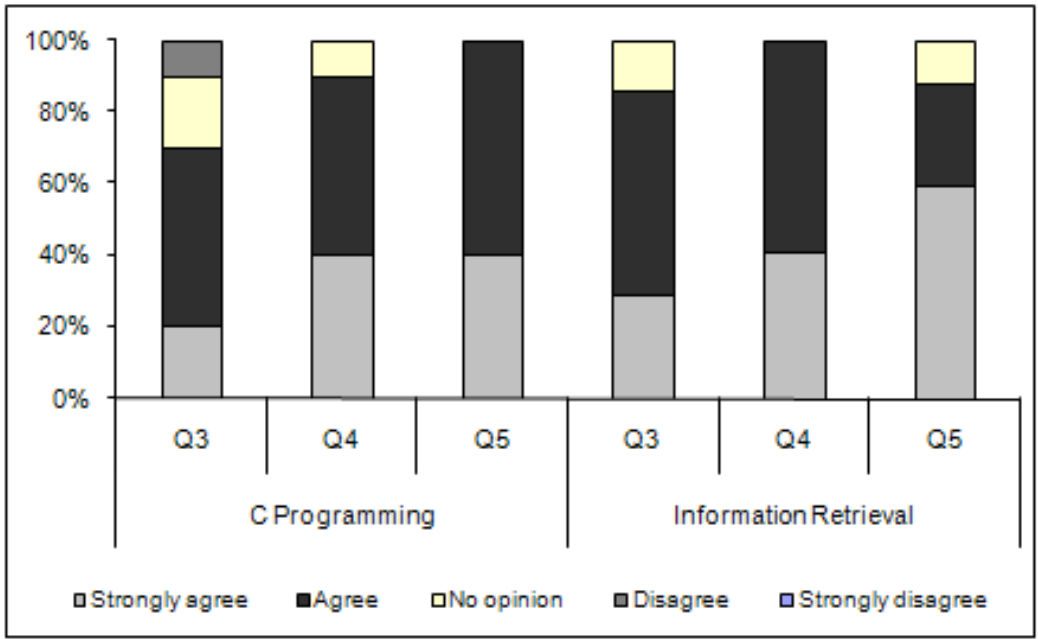

Figure 23. Students' attitude towards annotation-based SNS inside Web pages 


\section{Conclusion and Future Work}

AnnotatEd provides social navigation services as well as the ability for students to add annotations when browsing educational resources on the Web. AnnotatEd keeps track of page visiting actions and the annotation behavior of students and uses this information to offer social navigation support. Social navigation support is offered through the adaptive annotation of links inside Web pages. We evaluated the importance of providing the browsing option in addition to structured navigation options, such as map-based navigation or search-based navigation. Our results showed that browsing is used by the students at least as frequently as map-based navigation, and more frequently than searching. We also evaluated the effect of traffic- and annotation-based SNS on student decision making. Our results show that students access augmented links significantly more frequently than not annotated ones. Moreover, we assessed the importance of pages that had been annotated by students. The results suggest that pages with student annotations were more important to students. The subjective data analysis uncovered a highly positive student attitude towards the annotation functionality of the system and annotation-based SNS.

More extensive evaluation of traffic-based and annotated-based remains the most important goal of our future work. We would like to evaluate AnnotatEd system with a higher number of users as well as to explore in more details how the users interact with the system and its annotation and social navigation features. For example, do all users have equal chances to be the first to read a page before it receives any traffic-based annotation? Or, instead, the users can be split into "explorers", who read book pages ahead of their peers, and "followers", who tend to read pages rather late? Similarly, is the percentage of annotated pages comparable for different users or we can split users on "annotaters", who leave the majority of annotations, and "readers" who use the annotations but rarely leave any? In addition, using the eye-tracker we want to explore to what extent users pay attention to social visual cues. How much of user attention the visual cues attract? How frequently the users choose to follow visual cues, which they noticed and examined?

We would also like to improve our annotation interface by making the annotation process easier, in particular, by enabling pen-based annotation. We hypothesize that a more advanced annotation interface will encourage students to annotate more. Also, we want to expand the bookmarking feature of the system, which is currently very primitive, and to explore the value of navigation support based on the analysis of pages bookmarked by students. Finally, we would like to explore the use of AnnotatEd in other contexts and in conjunction with different information access systems.

\section{Acknowledgements}

This research is supported by the National Science Foundation under Grant No. 0447083 and the National Science Foundation graduate fellowship to the first author. 


\section{References}

R. Barrett and P.P. Maglio, 1999. Intermediaries: An approach to manipulating information streams. IBM Systems Journal, 38 (4), 629-641.

P. Bottoni, S. Levialdi, A. Labella, E. Panizzi, R. Trinchese and L. Gigli, 2006. MADCOW: a visual interface for annotating web pages. Working conference on Advanced visual interfaces (AVI'2006) ACM Press Venezia, Italy, 314-317.

S. Bradshaw and M. Light, 2007. Annotation consensus: implications for passage recommendation in scientific literature. 18th conference on Hypertext and hypermedia, HT '07 ACM Press, 209-216.

A.J.B. Brush, D. Bargeron, J. Grudin, A. Borning and A. Gupta, 2002. Supporting Interaction Outside of Class: Anchored Discussions vs. Discussion Boards. In: G. Stahl, ed. Proccedings of Computer Support for Collaborative Learning, CSCL 2002, Boulder, CO, 425-434.

P. Brusilovsky, 1996. Methods and techniques of adaptive hypermedia. User Modeling and User-Adapted Interaction, 6 (2-3), 87-129.

P. Brusilovsky, 2001. Adaptive hypermedia. User Modeling and User Adapted Interaction, 11 (1/2), 87-110.

P. Brusilovsky, G. Chavan and R. Farzan, 2004. Social adaptive navigation support for open corpus electronic textbooks. In: P. De Bra and W. Nejdl, eds., Third International Conference on Adaptive Hypermedia and Adaptive Web-Based Systems (AH'2004) Lecture Notes in Computer Science 3137, Springer-Verlag, Eindhoven, the Netherlands, 24-33.

P. Brusilovsky and N. Henze, 2007. Open corpus adaptive educational hypermedia. In: P. Brusilovsky, A. Kobsa and W. Neidl, eds. Open corpus adaptive educational hypermedia. Lecture Notes in Computer Science 4321. Berlin Heidelberg New York: Springer-Verlag,671-696.

J.J. Cadiz, A. Gupta and J. Grudin, 2000. Using Web annotations for asynchronous collaboration around documents. CSCW'00: Proceedings of the 2000 ACM conference on Computer supported cooperative work ACM Press, 309-318.

M. Claypool, P. Le, M. Wased and D. Brown, 2002. Implicit interest indicators. 6th International Conference on Intelligent User Interfaces ACM Press, Santa Fe, NM, 3340.

L. Denoue and L. Vignollet, 2001. Personal Information Organization using Web Annotations. In: W. Fowler and J. Hasebrook, eds., WebNet'2001, World Conference of the WWW and Internet AACE, Orlando, FL, 279-283.

A. Dieberger, P. Dourish, K. Höök, P. Resnick and A. Wexelblat, 2000. Social navigation: Techniques for building more usable systems. interactions, 7 (6), 36-45.

A. Dieberger and M. Guzdial, 2003. CoWeb - experiences with collaborative Web spaces. In: C. Lueg and D. Fisher, eds. CoWeb - experiences with collaborative Web spaces. New York: Springer-Verlag,155-166.

P. Dourish and M. Chalmers, 1994. Running out of space: Models of information navigation. In: G. Cockton, S.W. Draper and G.R.S. Weir, eds., HCI'94 Cambridge University Press, Glasgow.

R. Farzan and P. Brusilovsky, 2005. Social navigation support in E-Learning: What are real footprints. In: S.S. Anand and B. Mobasher, eds., IJCAI'05 Workshop on Intelligent 
Techniques for Web Personalization, Edinburgh, U.K., 49-56, also available at http://maya.cs.depaul.edu/ mobasher/itwp05/final/Paper7Farzan.pdf.

R. Farzan and P. Brusilovsky, 2005. Social navigation support through annotation-based group modeling. In: L. Ardissono, P. Brna and A. Mitrovic, eds., 10th International User Modeling Conference Lecture Notes in Artificial Intelligence 3538, Springer Verlag, Berlin, 463-472.

R. Farzan, M. Coyle, J. Freyne, P. Brusilovsky and B. Smyth, 2007. ASSIST: adaptive social support for information space traversal. 18th conference on Hypertext and hypermedia, HT '07 ACM Press, Manchester, UK, 199-208.

J. Freyne, R. Farzan, P. Brusilovsky, B. Smyth and M. Coyle, 2007. Collecting community wisdom: Integrating social search \& social navigation. International Conference on Intelligent User Interfaces, IUI 2007 ACM Press, Honolulu, HI, USA, 52-61.

Gibeo, Shared Web Annotation \& Content Aware Services, Gibeo, LLC, 2004, available online at

J. Kahan, Koivunen, E. Prud'hommeaux and R.R. Swick, 2002. Annotea: an open RDF infrastructure for shared Web annotations. Computer Networks, 39 (5), 589-608.

J. Kurhila, M. Miettinen, P. Nokelainen, P. Floréen and H. Tirri, 2003. Joint Annotation and Knowledge Building in Collaborative E-Learning. In: A. Rossett, ed. World Conference on E-Learning, E-Learn 2003 AACE, Phoenix, AZ, USA, 1487-1490.

J. Kurhila, M. Miettinen, P. Nokelainen and H. Tirri, 2002. EDUCO - A collaborative learning environment based on social navigation. In: P. De Bra, P. Brusilovsky and R. Conejo, eds., Second International Conference on Adaptive Hypermedia and Adaptive Web-Based Systems (AH'2002) Lecture Notes in Computer Science 2347, Málaga, Spain, 242-252.

P. Moody: WebPath: Sharable Personalized Guided Web Tours, Technical Report No. 98-09, Lotus (1998).

T. Noda, T. Fujimoto and H. Suzuki, 2004. WebAngel2: A browser add-on for utilizing existing Web resources as educational material. In: L. Cantoni and C. McLoughlin, eds., EDMEDIA'2004 - World Conference on Educational Multimedia, Hypermedia and Telecommunications AACE, Lugano, Switzerland, 688-692.

M. Pazzani, J. Muramatsu and D. Billsus, 1996. Syskill \& Webert: Identifying interesting Web sites. the Thirteen National Conference on Artificial Intelligence, AAAI'96 AAAI Press /MIT Press, Portland, OR, 54-61.

P.-L.P. Rau, S.-H. Chen and Y.-T. Chin, 2004. Developing web annotation tools for learners and instructors. Interacting with Computers, 16 (2), 163-181.

E.S. Sandvad, G. K., S. L. and K. J.L., 2001. A Metro Map Metaphor for Guided Tours on the Web: the Webvise Guided Tour System. Tenth International World Wide Web Conference, Hong Kong, 326-333.

B.N. Schilit, G. Golovchinsky and M.N. Price, 1998. Beyong paper: Supporting Active reading with free form digital ink annotations. In: C.-M. Karat, A. Lund, J. Coutaz and J. Karat, eds., $\mathrm{CHI}^{\prime} 98$ ACM Press, Los Angeles, CA.

F. Shipman, R. Furuta, D. Brenner, C. Chung and H. H., 2000. Guided Paths through Web-Based Collections: Design, Experiences, and Adaptations. Journal of the American Society for Information Science, 51 (3), 260-272.

A. Wexelblat and P. Mayes, 1999. Footprints: History-rich tools for information foraging. $A C M$ Conference on Human-Computer Interaction (CHI'99), Pittsburgh, PA, 270-277. 
P.G. Wojahn, C.M. Neuwirth and B. Bullock, 1998. Effects of interfaces for annotation on communication in a collaborative task. CHI98: Human Factors in Computing Systems ACM Press, Los Angeles, CA, USA, 456-463.

C. Xin and G. Glass, 2005. Enhancing Online Discussion through Web Annotation. In: G. Richards, ed. World Conference on E-Learning, E-Learn 2005 AACE, Vancouver, Canada, 3212-3217.

M. Yanagisawa, K. Ito and K. Akahori, 2004. Web Memo: A WWW Browser for Learning with Support for Writing Notes in Web Material. International Conference on Computers in Education, ICCE 2004 AACE, Melbourne, Australia, 2069-2075.

K.-P. Yee, 2002. CritLink: Advanced Hyperlinks Enable Public Annotation on the Web. ACM Conference on Computer-Supported Cooperative Work, CSCW 2002 (Demonstration abstract). 
Table 1 - General usage statistics

Table Caption

Table 2 - Quantity of anonymous versus signed annotations

Table 3 - Type of anonymous versus signed annotations

Table 4 - Time spent on pages accessed through the map compared to time spent when browsing (in seconds)

Table 5 - Average number of clicks for pages with and without traffic/annotation-based SNS

Table 6 - Average access to pages with and without annotation, before the occurrence of student annotation 
Figure Caption.

Figure 1. Sample page in AnnotatEd

Figure 2. AnnotatEd in the context of the Knowledge Sea II System

Figure 3. Navigational Cues

Figure 4. Visual icons representing the different magnitudes of group visiting activity

Figure 5. The use of AnnotatEd in the ASSIST-ACM System

Figure 6. Background color levels representing the magnitude of group visiting activity

Figure 7. Computation of real visit based on TSR

Figure 8. Example of traffic levels, according to number of clicks

Figure 9. Visual cues representing different levels of individual visiting activity

Figure 10. The Annotation Interface (Stage II)

Figure 11. Background color levels representing the magnitude of group annotation activity

Figure 12. The Annotation Interface (Stage III)

Figure 13. Stage I: Subjective questions evaluating annotation features

Figure 14. Stage I - Subjective opinions of students about the annotation features

Figure 15. Effect of the presence of public annotation on student visits

Figure 16. The length of anonymous versus signed annotations

Figure 17. Stage II - Subjective question evaluating annotation features and annotation-based social navigation support

Figure 18. Stage II - Subjective opinions of students about the annotation features

Figure 19 - Percentage of access from each method

Figure 20. Effect of the presence of public annotation on student visits

Figure 21. Stage III - Subjective question evaluating annotation features and annotation-based social navigation support

Figure 22. Student attitude towards the annotation functionality of the system

Figure 23. Students' attitude towards annotation-based SNS inside Web pages 